\title{
Optimal matrices of partitions and an application to Souslin trees
}

\author{
by
}

\author{
Gido Scharfenberger-Fabian (Greifswald)
}

\begin{abstract}
The basic result of this note is a statement about the existence of families of partitions of the set of natural numbers with some useful properties, the $n$-optimal matrices of partitions. We use this to improve a decomposition result for strongly homogeneous Souslin trees. The latter is in turn applied to separate strong notions of rigidity of Souslin trees, thereby answering a considerable portion of a question of Fuchs and Hamkins.
\end{abstract}

Introduction. In many models of set theory, Souslin trees offer a variety of different homogeneity or rigidity properties. Probably the most prominent homogeneity property for Souslin trees is strong homogeneity (cf. Section 2.2 for the definition) which implies that the tree is in a certain sense minimal with respect to its automorphism group. On the other hand, a great number of rigidity notions (i.e. absence of nontrivial automorphisms) for Souslin trees and an array of implications between most of them are known. In this paper, which resulted out of a part of the author's $\mathrm{PhD}$ thesis [SF08], we present some interrelations between the class of strongly homogeneous Souslin trees and that of free trees, the latter consisting of those Souslin trees which have the strongest known rigidity properties.

The key result which leads to these correspondences is a certain method for decomposing a strongly homogeneous Souslin tree into $n$ free factors (Theorem 2.4, which is a strengthening of a known though unpublished result). This decomposition uses an elementary, but apparently new combinatorial tool, an $n$-optimal matrix of partitions, which we introduce in the first section. As will be seen in Section 2, there are several ways to decompose a strongly homogeneous Souslin tree into $n$ free trees. But the construction we give using an $n$-optimal matrix of partitions enables us to prove strong consequences about the behaviour of the factors which are finally used in the

2010 Mathematics Subject Classification: Primary 03E05; Secondary 05A18. Key words and phrases: Souslin trees, homogeneity, rigidity, partition. 
third section to separate certain notions of parametrized rigidity for Souslin trees (which are all weakenings of freeness) in Corollaries 3.5 and 3.7.

A few words on the structure of the paper and the assumed background which differs strongly from section to section. The first section is about the very elementary notion of $n$-optimal matrices of partitions and does not assume any prerequisites. The other two sections treat Souslin trees and their structural properties. In Section 2 we review strong homogeneity and freeness for Souslin trees and prove two decomposition theorems for strongly homogeneous Souslin trees. The final section collects several rigidity notions for Souslin trees (most of them taken from [FH09]) and gives the aforementioned separation results. Some definitions and proofs in Section 3 refer to the technique of forcing which we do not review here. And even though we give the necessary definitions concerning Souslin trees at the beginning of Section 2, some acquaintance with this subject will certainly enhance the reader's understanding of the constructions in Section 2 (very good references, also on forcing, are, e.g., [DJ74, Kun80, Jec03]). Anyway, we have made an effort to write a paper that is accessible to an audience exceeding the circle of experts on Souslin trees.

1. Optimal matrices of partitions. The main idea is as follows: Consider an infinite matrix with $\omega$ rows and $n$ columns where $n$ is a natural number larger than 1:

$$
\left(\begin{array}{ccccc}
P_{0,0} & \ldots & P_{0, m} & \ldots & P_{0, n-1} \\
\vdots & \vdots & \vdots & \vdots & \vdots \\
P_{k, 0} & \ldots & P_{k, m} & \ldots & P_{k, n-1} \\
\vdots & \vdots & \vdots & \vdots & \vdots
\end{array}\right)
$$

Suppose that the entries of this matrix are partitions of the set $\omega$ of natural numbers. We want to choose these partitions in such a way that (i) we get an infinite set whenever we intersect a finite family of subsets of $\omega$ coming from (distinct) partitions of a single column and (ii) we get a singleton whenever we intersect $n$ sets belonging to partitions each coming from different columns. In the following definition the latter requirement is stated in a slightly stronger form: we want to obtain a singleton whenever we intersect $n$ sets not all coming from the same column. The construction in the proof of Lemma 1.2 actually yields matrices that satisfy this stronger condition, and we will use it in the proof of Proposition 2.5 to derive an additional result.

Definition 1.1. For $n \in \omega$, an $n$-optimal matrix of partitions is a family $\left(P_{k, m} \mid k \in \omega, m<n\right)$ of infinite partitions $P_{k, m}=\left(a_{i}^{k, m} \mid i \in \omega\right)$ of $\omega$ with the following properties. 
(i) Columnwise agreement: For all $m<n$ and all $i: k \rightarrow \omega$ where $k \in \omega$, the intersection $\bigcap_{\ell<k} a_{i(\ell)}^{\ell, m}$ is infinite.

(ii) $n$-optimality: For all maps $(i, k, m): n \rightarrow \omega \times \omega \times n$ with $(k(j), m(j))$ $\neq(k(\ell), m(\ell))$ for all $j<\ell<n$ and $m(j) \neq m(\ell)$ for at least one pair $j, \ell<n$ the intersection

$$
\bigcap_{j<n} a_{i(j)}^{k(j), m(j)} \quad \text { is a singleton. }
$$

Note that if in (ii) the domain of $(i, k, m)$ is restricted to a proper subset of $n$, i.e., if we intersect over less than $n$ sets, then the corresponding intersection has to be infinite as well.

LEMma 1.2. There is an n-optimal matrix of partitions for every natural number $n>1$.

Proof. To start we fix a bijective enumeration $h=\left(h_{0}, \ldots, h_{n-1}\right): \omega \rightarrow$ $\omega^{n}$ and define $a_{i}^{0, m}$ to be the preimage of $i$ under $h_{m}$. Let $P_{0, m}:=\left\{a_{i}^{0, m} \mid\right.$ $i \in \omega\}$.

The rest of the construction consists of a three-fold recursion. The outer loop is indexed by $(k, m) \in \omega \times n$, and goes row by row, from left to right. One could also say that the progression of the indices follows the lexicographic order of $\omega \times n$, i.e., $m$ grows up to $n-1$ and then drops down to 0 while $k$ increases to $k+1$. (The first $n$ stages of the outer loop, where $k=0$, have been included in the recursive anchor in the first line of the proof.)

The inner recursion loops are common $\omega$-recursions. In each stage of the middle one we define one element $a_{i}^{k, m}$ of the partition $P_{k, m}$, and the innermost consists of a choice procedure for the elements of that set $a_{i}^{k, m}$.

So assume that the partitions $P_{\ell, m}=\left\{a_{i}^{\ell, m} \mid i \in \omega\right\}$ have already been defined for $(\ell, m)<_{\text {lex }}(k, n)$ and also the $i$ first sets $a_{0}^{k, m}=a_{0}, \ldots, a_{i-1}^{k, m}=$ $a_{i-1}$ of $P_{k, m}$ have been fixed. Assume also that the family constructed so far has the properties (i) and (ii) from Definition 1.1. We inductively choose three sequences $x_{\ell}, y_{\ell}$ and $z_{\ell}$ of members of $\omega \backslash \bigcup_{h<i} a_{h}$ and afterwards set $a_{i}:=\left\{x_{\ell}, y_{\ell} \mid \ell \in \omega\right\}$. The members of the $x$ sequence will make the matrix satisfy columnwise agreement (requirement (i)) while the $y_{\ell}$ guarantee that the intersections for (ii) ( $n$-optimality) are non-empty. The elements $z_{\ell}$ go back to the stack and build the pool for the construction of the further members of $P_{k, m}$.

We need the objects $f, b, I, c, \tau$ and $d$ below for book-keeping and assume that they have been fixed at the start of the definition of the members of the partition $P_{m, k}$, before the construction of $a_{0}$. 
Let $f: \omega \rightarrow{ }^{k} \omega$ be onto and $\aleph_{0}$-to-1 and set, for $\ell \in \omega$,

$$
b(\ell):=\bigcap_{j<k} a_{f(\ell)(j)}^{j, m} .
$$

These sets $b(\ell)$ have to be met by $a_{i}$ infinitely many times. So we will choose $x_{\ell}$ from $b(\ell)$. Let $I$ be the set of subsets $\sigma$ of $(\omega \times k \times n) \cup(\omega \times(k+1) \times m)$ of cardinality $n-1$ such that $\operatorname{pr}_{2,3}\left\lceil\sigma\right.$ is injective and $\operatorname{pr}_{3}\lceil\sigma$ is not equal to the constant function whose only value is $m$, where $\mathrm{pr}_{3}$ and $\mathrm{pr}_{2,3}$ are the projections to the third and to the second and third component of a triple respectively. So if $\sigma \in I$ then the elements of $\sigma$ are indices for $n-1$ members of pairwise distinct partitions and and at least one of these partitions lies outside of the $m$ th column of the $n$-optimal matrix. For $\sigma \in I$ let

$$
c(\sigma):=\bigcap_{(j, p, q) \in \sigma} a_{j}^{p, q}
$$

and note that this set is infinite, because if $P_{r, s}$ is a partition which is not involved in $\sigma$ then $c(\sigma)$ meets every element of $P_{r, s}$ in exactly one natural number by $n$-optimality.

Also the sets $c(\sigma)$ have to be met by $a_{i}$. So we fix a bijective enumeration $\tau$ of $I$ and choose $y_{\ell}$ from $c(\tau(\ell))$ unless that set has already been hit by earlier members of $a_{i}$.

Condition (ii) imposes that each set $c(\sigma)$ be met in only one element. So once the intersection of $a_{i}$ and $c(\sigma)$ is non-empty, that particular set $c(\sigma)$ has to be avoided in later choices of members of $a_{i}$. We thus define for every natural number $x$ the set

$$
d(x):=\bigcup\{c(\sigma) \mid \sigma \in I \text { and } x \in c(\sigma)\}
$$

and choose $x_{\ell}$ and $y_{\ell}$ from outside $\bigcup_{j<\ell} d\left(x_{j}\right) \cup d\left(y_{j}\right)$.

We now turn to the formal definition of our three sequences $x_{\ell}, y_{\ell}$ and $z_{\ell}$ and argue afterwards why these choices are always possible. Set $e=\bigcup_{h<i} a_{h}$ and let inductively

$$
\begin{aligned}
& x_{\ell}:=\min \left(b(\ell) \backslash\left(e \cup \bigcup_{j<\ell}\left(d\left(x_{j}\right) \cup d\left(y_{j}\right) \cup\left\{z_{j}\right\}\right)\right)\right), \\
& y_{\ell}:= \begin{cases}x_{\ell}, & c(\tau(\ell)) \cap \bigcup_{j<\ell}\left\{x_{j}, y_{j}, x_{j+1}\right\} \neq \emptyset, \\
\min c(\tau(\ell)) \backslash\left(e \cup\left\{z_{0}, \ldots, z_{\ell-1}\right\}\right), & \text { otherwise, }\end{cases} \\
& z_{\ell}:=\min \left(b(\ell) \backslash\left(e \cup \bigcup_{j<\ell}\left\{x_{j}, y_{j}, z_{j}, x_{j+1}, y_{j+1}\right\}\right)\right) .
\end{aligned}
$$

We finally show that this construction does not break down, i.e., the sets from which $x_{\ell}$ or $z_{\ell}$ are picked are non-empty for all $\ell$. (A variation shows that the choice $y_{\ell}$ is always possible, as well.) The argument splits depending on whether $k$ is less than or at least $n-1$. So let $k<n-1$ and $\ell \in \omega$, 
set $\rho=\rho(\ell)=\{(f(\ell)(j), j, m): j<k\}$ and fix $\sigma \in I$ such that $\rho \subset \sigma$, say $\sigma=\rho \cup\left\{\left(j_{1}, p_{1}, q_{1}\right), \ldots,\left(j_{r}, p_{r}, q_{r}\right)\right\}$ for $r=n-1-k$. Now let

$$
F:=F(\ell, \sigma):=\left\{\left\{\left(g_{1}, p_{1}, q_{1}\right), \ldots,\left(g_{r}, p_{r}, q_{r}\right)\right\}:\left(g_{1}, \ldots, g_{r}\right) \in \omega^{r}\right\}
$$

and note that $b(\ell)$ is the disjoint union of the family $\{c(\rho \cup \tau): \tau \in F\}$ :

$$
b(\ell)=\bigcup_{\tau \in F}^{\cdot} c(\rho \cup \tau) .
$$

In order to prove that we can always choose our new element of the $x$-, $y$ or $z$-sequence, we first state two consequences of $n$-optimality:

(1) For every $\tau \in F$ and $h<i$ the intersection $c(\rho \cup \tau) \cap a_{h}$ is a singleton.

(2) Given any $\sigma^{\prime} \in I$, for every $\tau \in F$ with $\sigma^{\prime} \neq \rho \cup \tau$ the intersection $c(\rho \cup \tau) \cap c\left(\sigma^{\prime}\right)$ has at most one element.

Now note that in order to define $x_{\ell}$ or $z_{\ell}$, we subtract only finitely many sets of the form $a_{h}$ or $c\left(\sigma^{\prime}\right)$ from $b(\ell)$. So in any case, infinitely many natural numbers remain in the pool.

Next let $k \geq n-1$. Then any set of the form $c(\sigma)$ meets $b(\ell)$ in at most one member by a direct application of $n$-optimality. Furthermore the $z$-part of the construction implies by induction on $i$ that $b(\ell) \backslash e=b(\ell) \backslash \bigcup_{h<i} a_{h}$ is an infinite set.

We leave to the reader the verification that this construction indeed yields an $n$-optimal matrix of partitions.

There is also a proof of Lemma 1.2 which uses a more sophisticated forcing style argument in the construction of the partition $P_{k, m}$, but since it does not significantly decrease the length of the proof we stuck to the elementary recursive construction given above.

\section{Strongly homogeneous and free Souslin trees}

2.1. Preliminaries on Souslin trees. A tree is a partial order $\left(T,<_{T}\right)$ where for all $t \in T$ the set of predecessors $\left\{s \mid s<_{T} t\right\}$ is well-ordered by $<_{T}$. The elements of a tree are called nodes. For a node $t \in T$ we let $\operatorname{succ}(t)$ be the set of $t$ 's immediate successors. The height of the node $t$ in $T$ is the order type of the set of its predecessors under the ordering of $T$, $\mathrm{ht}_{T}(t):=\operatorname{ot}\left(\left\{s \mid s<_{T} t\right\},<_{T}\right)$. For an ordinal $\alpha$ we let $T_{\alpha}$ denote the set of nodes of $T$ with height $\alpha$. If $\operatorname{ht}_{T}(s)>\alpha$ we let $s\lceil\alpha$ be the unique predecessor of $s$ in level $\alpha$.

The height of a tree $T$, ht $T$, is the minimal ordinal $\alpha$ such that $T_{\alpha}$ is empty. An antichain is a set of pairwise incomparable nodes of $T$, so for $\alpha<\operatorname{ht} T$, the level $T_{\alpha}$ is an antichain of $T$.

Nodes that do not have $<_{T}$-successors are called leaves, and $T$ is called $\kappa$-splitting or $\kappa$-branching, $\kappa$ a cardinal, if all nodes of $T$ have exactly $\kappa$ immediate successors, except for the leaves. 
A branch is a subset $b$ of $T$ that is linearly ordered by $<_{T}$ and downwards closed, i.e. if $s<_{T} t \in b$ then $s \in b$. Under the notion of a normal tree we subsume the following four conditions:

(a) there is a single minimal node called the root;

(b) each node $s$ with ht $(s)+1<$ ht $T$ has at least two immediate successors;

(c) each node has successors in every higher non-empty level;

(d) branches of limit length have unique limits (if they are extended in the tree), i.e., if $s, t$ are nodes of $T$ of limit height whose sets of predecessors coincide, then $s=t$.

Note that by condition (c), leaves can only appear in the top level of a normal tree.

For a node $t \in T$ we denote by $T(t)$ the set $\left\{s \in T: t \leq_{T} s\right\}$ of nodes above (and including) $t$, which becomes a tree when equipped with the ordering inherited from $T$. A tree $T$ is said to be homogeneous if for all pairs $s, t \in T$ of the same height there is a tree isomorphism (of partial orders) between $T(s)$ and $T(t)$, the trees of nodes in $T$ above $s$ and $t$ respectively. For many classes of trees, such as Souslin trees, this is equivalent to the condition that for each pair $s, t \in T$ of nodes of the same height there is an automorphism of $T$ mapping $s$ to $t$. A tree is rigid if it does not admit any non-trivial automorphism.

We will consider two operations on the class of trees: sum and product. Given trees $\left(T^{i},<_{i}\right)$ for $i \in I$, the tree sum of this family, denoted by $\bigoplus_{i \in I} T^{i}$, is the disjoint union of the sets $T^{i}$ plus a common root $r \notin \bigcup T^{i}$. The tree order $<$ on $\bigoplus T^{i}$ is given by the (disjoint) union of the tree orders of the summands as well as the relation $r<t$ for all $t \in \bigcup T^{i}$. The height of $\bigoplus T^{i}$ is given by the ordinal $1+\sup \left\{\right.$ ht $\left.T^{i}: i \in I\right\}$.

Let now all trees $T^{i}$ be of height $\mu$. The tree product $\bigotimes_{i \in I} T^{i}$ over the family $\left(T^{i}\right)_{i \in I}$ is given by the union over the cartesian products of the levels $T_{\alpha}^{i}$ :

$$
\bigotimes_{i \in I} T^{i}:=\bigcup_{\alpha<\mu} \prod_{i \in I} T_{\alpha}^{i}
$$

The product tree order is simply the conjunction of the relations $<_{i}$.

In order to make a decomposition of a tree into a product feasible we also introduce the notion of a nice tree equivalence relation. Let $T$ be a normal and $\aleph_{0}$-splitting tree and $\equiv$ an equivalence relation on $T$. Then we say that $\equiv$ is a nice tree equivalence relation (nice t.e.r.) if $\equiv$ respects levels (i.e., it refines $T \otimes T$ ), is compatible with the tree order (i.e., ht $(s)=\operatorname{ht}(r)$ and $s<t \equiv u>r$ imply $s \equiv r)$, the quotient partial order $T / \equiv$ of $\equiv$-classes 
ordered by the inherited partial order, i.e.

$$
[s]<\equiv[t] \Leftrightarrow s<t,
$$

is a normal and $\aleph_{0}$-splitting tree and the relation is nice, by which we mean that for all triples of nodes $s, r, t$ such that $s \equiv r$ and $t$ is above $s$ there is a node $u \equiv t$ with $u$ above $r$. Another way to formulate this last property is that "niceness" associates to each branch $b$ through $T$ a subtree $T_{\equiv}^{b}:=$ $\bigcup_{s \in b} s / \equiv$ of $T$ and requires that it satisfies point (c) in our definition of normal trees, i.e., every node $t \in T_{\equiv}^{b}$ has successors in every higher level of $T_{\equiv}^{b}$.

Now consider the case that a tree $T$ carries nice tree equivalence relations $\equiv_{i}$ for $i<n$ such that for every level $\alpha$ and every $n$-sequence of equivalence classes $c_{i} \in\left(T / \equiv_{i}\right)_{\alpha}, i<n$, the intersection $\bigcap c_{i}$ is a singleton $\{t\}$ with $t \in T_{\alpha}$. Then we have a natural isomorphism between the tree $T$ and the product of its quotient trees, $\bigotimes_{i<n} T / \equiv_{i}$ given by $t \mapsto\left(t / \equiv_{i}: i<n\right)$.

We finally come to Souslin trees. In general, a Souslin tree is a tree $T$ of height $\omega_{1}$ such that every family of pairwise incomparable nodes and also every branch of $T$ is at most countable. Unless stated otherwise, we will only consider normal and $\aleph_{0}$-splitting Souslin trees. In this case the sole absence of uncountable antichains - referred to as the countable chain condition (c.c.c.) - already implies that the tree has no cofinal branch. A main elementary feature of Souslin trees is that their square is no longer Souslin: $T \otimes T$ violates the c.c.c. for every Souslin tree $T$.

The naming Souslin of these trees stems from their tight connection to the famous question of Mikhail Yakovlevich Souslin that was published as Problème 3) on page 223 of the first issue of Fundamenta Mathematicae in 1920 (cf. also [DJ74] or [Kun80, II.4]; the latter transliterates the name as Suslin). It is well known that the existence of Souslin trees is independent of ZFC, so whenever we assume that there is some Souslin tree, we make an extra assumption beyond the realm of standard set theory.

2.2. Strongly homogeneous and free trees. We take a closer look at two classes of Souslin trees, that are widely known among set-theorists, although often under different names, of which we try to state as many as possible.

Strongly homogeneous Souslin trees occur quite often in set-theoretic literature. In [LT02] they are called coherent Souslin trees and play a central role in the solution of Katětov's Problem on the metrizability of certain compact spaces. Shelah and Zapletal show in [SZ99, Theorem 4.12] that Todorčević's term for a Souslin tree in one Cohen real is strongly homogeneous, Larson gives a direct $\diamond$-construction ([Lar99, Lemma 1.2]), and also Jensen's construction (under the same hypothesis) of a 2-splitting, homo- 
geneous tree, as carried out in [DJ74, Chapter IV], is easily seen to yield a strongly homogeneous tree.

Definition 2.1. A Souslin tree $T$ is called strongly homogeneous if there is a family $\left(\psi_{s t} \mid s, t \in T\right.$, ht $\left.s=\mathrm{ht} t\right)$ which has the following properties:

(1) $\psi_{s t}$ is an isomorphism between the tree $T(s)$ of nodes above $s$ and the tree $T(t)$ of nodes above $t$ and $\psi_{s s}$ is the identity.

(2) (Commutativity) For all nodes $r, s, t$ of the same level of $T$ we have $\psi_{r t}=\psi_{s t} \circ \psi_{r s}$.

(3) (Coherence) For nodes $r, s$ from the same level, $t$ above $r$ and $u=$ $\psi_{r s}(t)$ we require that $\psi_{t u}$ is the restriction of $\psi_{r s}$ to the tree $T(t)$ $\subset T(r)$.

(4) (Transitivity) If $t$ and $u$ are nodes on the same limit level $T_{\alpha}$, then there is a level $T_{\gamma}$ below such that for the corresponding predecessors $r$ of $t$ and $s$ of $u$ we have $\psi_{r s}(t)=u$.

Some authors call such a family of tree isomorphisms associated to a strongly homogeneous tree a coherent family.

Given any homogeneous tree, it is easy to define a family on $T$ with the properties (1)-(3) above. The crucial property of a coherent family is that of transitivity, which means that every limit level is a minimal extension of the initial segment below with respect to the coherent family on that initial segment. Also the automorphism group of a strongly homogeneous Souslin tree $T$ is in a sense minimal, as shown in the following proposition.

Proposition 2.2. Every automorphism $\varphi$ of a strongly homogeneous Souslin tree $T$ is eventually equal to the union of a subset of the coherent family $\left(\psi_{s t}\right)$, i.e., there is a countable ordinal $\alpha$ such that for all nodes $t$ of height greater than $\alpha$ we have

$$
\varphi(t)=\psi_{t\lceil\alpha, \varphi(t\lceil\alpha)}(t) .
$$

This implies that the automorphism group of such a tree has exactly $2^{\aleph_{0}}$ elements.

Proof. It suffices to show that above every node $r \in T$ there is a node $s$ such that all $t>s$ are mapped by the automorphism $\varphi$ according to the rule stated above with $\alpha=\operatorname{ht}(s)$.

To reach a statement contradicting the transitivity of the family $\left(\psi_{s t}\right)$, we assume that there is a node $r \in T$ such that for each successor $s$ of $r$ there is a node $t \geq s$ such that $\varphi(t) \neq \psi_{s \varphi(s)}(t)$. We can inductively choose an increasing sequence of ordinals $\alpha_{n}$ such that for all nodes $t \in T_{\alpha_{n+1}}$ we have

$$
\varphi(t) \neq \psi_{t\left\lceil\alpha _ { n } \varphi \left( t\left\lceil\alpha_{n}\right)\right.\right.}(t) .
$$


Let $\alpha$ be the supremum of the $\alpha_{n}$ and pick any node $t \in T_{\alpha}$. Since $\alpha$ is a limit ordinal and by transitivity of the coherent family we find an $n \in \omega$ such that $\varphi(t)=\psi_{t\left\lceil\alpha_{n} \varphi\left(t\left\lceil\alpha_{n}\right)\right.\right.}(t)$, which is of course impossible by the choice of the $\alpha_{n}$.

Now we come to free trees. Also this property has several different names, e.g. full (Jensen, Todorčević [Jen, Tod84]) or "Souslin and all derived trees Souslin" (Abraham and Shelah, AS85, AS93]). In the context of [FH09] (cf. Section 3.1 of the present article) free trees could also be called " $<\omega$-fold Souslin off the generic branch".

Definition 2.3. A normal tree $T$ of height $\omega_{1}$ is free if for every finite (and non-empty) set of nodes $s_{0}, \ldots, s_{n}$ of $T$ of the same height, the tree product $\bigotimes_{i=0}^{n} T\left(s_{i}\right)$ satisfies the c.c.c.

Free trees are easily seen to be rigid Souslin trees as the product of two isomorphic relative trees $T(s)$ and $T(t)$ would clearly not be Souslin. In Section 3 we will also consider weaker, parametrized forms of freeness.

2.3. Decompositions of strongly homogeneous Souslin trees. We now come to the key result of this paper. The following theorem is stated in [SZ99, p. 246] in the case $n=2$ without proof. Larson gives the construction of a single free subalgebra of a strongly homogeneous Souslin algebra in terms of trees in the proof of Theorem 8.5 in his paper [Lar99]. Some ideas in the following proof are borrowed from that construction.

THEOREM 2.4. For every natural number $n>1$ and every $\aleph_{0}$-branching, strongly homogeneous Souslin tree $T$ there are free Souslin trees $S_{0}, \ldots, S_{n-1}$ such that $T \cong \bigotimes_{m<n} S_{m}$.

Proof. Let $T$ be a strongly homogeneous Souslin tree and denote by $\psi_{s, t}$ the members of the coherent family of $T$. We inductively (level by level) define $n$ nice t.e.r.s $\equiv_{0}, \ldots, \equiv_{n-1}$ with the following properties:

- $T / \equiv_{m}$ is a free Souslin tree for $m<n$.

- For any sequence $\left(s_{0}, \ldots, s_{n-1}\right) \in T_{\alpha}$ the intersection of the classes $s_{m} / \equiv_{m}$ for $m<n$ is a singleton: $\bigcap_{m<n}\left(s_{m} / \equiv_{m}\right)=\{r\}$ for some $r \in T_{\alpha}$.

The second claim entails the existence of the isomorphism between $T$ and $\bigotimes_{m<n} T / \equiv_{m}$.

Let $\left(P_{k, m}: m<n, k \in \omega\right)$ be an $n$-optimal matrix of partitions, where we view each $P_{k, m}$ as enumerated by $a_{i}^{k, m}, i \in \omega$. In order to define t.e.r.s we transfer the whole matrix of the $P_{k, m}$ to every set $\operatorname{succ}(s)$ for $s \in T$ in a coherent way: Choose for every $\alpha<\omega_{1}$ an anchor node $r_{\alpha} \in T_{\alpha}$ and a bijection $\sigma_{\alpha}: \omega \rightarrow \operatorname{succ}\left(r_{\alpha}\right)$, and define for $s \in T_{\alpha}$ and all indices $i, k, m$ the 
sets

$$
a_{i}^{k, m}(s):=\left(\psi_{r_{\alpha}, s} \circ \sigma_{\alpha}\right)^{\prime \prime} a_{i}^{k, m} .
$$

Then clearly for every $s \in T, k \in \omega$ and $m<n$, the set $P_{k, m}(s):=\left\{a_{i}^{k, m}(s) \mid\right.$ $i \in \omega\}$ forms a partition of $\operatorname{succ}(s)$, and these partitions are linked to each other by the coherent family in a coherent way, i.e., $\psi_{s, t}$ transfers $P_{k, m}(s)$ to $P_{k, m}(t)$.

Fix $m<n$ in order to define $\equiv_{m}$ on $T$ by recursion on height. We will also enumerate the $\equiv_{m}$-classes of each level in order type $\omega$, i.e., we will fix an onto mapping $h: T \rightarrow \omega$ such that for $s, t \in T_{\alpha}$ we have $s \equiv_{m} t$ if and only if $h(s)=h(t)$.

Choose $P_{0, m}$ (root) as the partition of the set $T_{1}=\operatorname{succ}($ root) and let $\equiv_{m}$ on level $T_{1}$ be the equivalence relation with classes $a_{i}^{0, m}$ (root) for $i \in \omega$. Let $h$ (root) $=0$ and choose $h$ on $T_{1}$ in such a way that nodes $s$ and $t$ are $\equiv_{m}$-equivalent just in case that their $h$-values coincide.

Next we consider the case where $\alpha$ is a successor ordinal, $\alpha=\gamma+1$ for some $\gamma<\omega_{1}$. Let $s, t \in T_{\alpha}$ and let $s^{-}<_{T} s$ and $t^{-}<_{T} t$ be their direct predecessors on level $\gamma$. We let $s \equiv_{m} t$ if and only if their direct predecessors are $\equiv_{m}$-equivalent, $s^{-} \equiv_{m} t^{-}$(so in particular $h\left(s^{-}, m\right)=h\left(t^{-}, m\right)$ ), and if there is $i \in \omega$ such that

$$
s \in a_{i}^{h\left(s^{-}\right), m}\left(s^{-}\right) \text {and } t \in a_{i}^{h\left(t^{-}\right), m}\left(t^{-}\right) .
$$

In words, the $\equiv_{m}$-equivalence of the direct predecessors gives us a natural number $h\left(s^{-}\right)$and we apply $P_{h\left(s^{-}\right), m}$ on level $\alpha$ to decide whether or not $s$ and $t$ are $\equiv_{m}$-equivalent. Extend $h$ to level $T_{\alpha}$ as described above.

On limit stages $\lambda$ the relation $\equiv_{m}$ is already determined by its behaviour below, and we choose the $h\left\lceil T_{\lambda}\right.$ once more in any way such that $h(s)=h(t)$ is equivalent to $\equiv_{m}$-equivalence for nodes $s, t \in T_{\lambda}$.

Having finished the construction of the relation $\equiv_{m}$, we show that it produces a nice t.e.r., where all properties but niceness follow rather easily from the construction. So we only deduce niceness. Letting $s \equiv_{m} r$ on level $\alpha$ and $t$ above $s$ we claim that $\psi_{s, r}(t) \equiv_{m} t$ and show this by induction on the height of $t$ above $s$. For successor stages the claim follows directly from the construction and the inductive hypothesis, since the relevant partition $P_{j, m}$ is transferred via $\psi_{s, r}$ by the coherence of the coherent family. The limit case follows directly from the inductive assumption. (This property of $\equiv_{m}$, that $\equiv_{m}$-equivalence lifts from $s$ and $r$ to preimages and images under $\psi_{s, r}$, will be used again in the proof of the Claim below.)

It remains to prove the two properties stated before the construction. We start with the freeness of $T / \equiv_{m}$. Let $s_{0}, \ldots, s_{k-1}$ be pairwise non-mequivalent nodes of the same height $\alpha$ for some natural number $k$. We write $S_{i}$ for $\left(T / \equiv_{m}\right)\left(s_{i} / \equiv_{m}\right)$ and try to find for every antichain $A$ of $\bigotimes_{i<k} S_{i}$ an 
antichain $B$ of $T$ of the same cardinality. This would prove that $T / \equiv_{m}$ is a free tree. We get a hint about where to look for the members of such an antichain $B$ from the following

Claim. Fix $m<n$ and pairwise non-m-equivalent nodes $s_{0}, \ldots, s_{k-1}$ $\in T_{\alpha}$. For any sequence $\left(t_{0}, \ldots, t_{k-1}\right)$ of nodes in $T_{\beta}$, with $\alpha<\beta$ and $s_{i}<t_{i}$ for $i<k$, the intersection of the classes $t_{i} / \equiv_{m} \cap T\left(s_{i}\right)$ above the nodes $s_{i}$, shifted above $s_{0}$ by $\psi_{s_{i}, s_{0}}$, i.e., the set

$$
\bigcap_{i<k} \psi_{s_{i}, s_{0}}{ }^{\prime \prime}\left(t_{i} / \equiv_{m}\right)
$$

is infinite and therefore non-empty.

Proof of the Claim. By induction on the height $\beta$ of the nodes $t_{i}$, starting with $\beta=\alpha+1$. In this minimal case we have $t_{i}^{-}=s_{i}$. So the sets $\psi_{s_{i}, s_{0}}{ }^{\prime \prime}\left(t_{i} / \equiv_{m}\right)$ belong to distinct partitions $P_{h\left(s_{i}\right), m}\left(s_{0}\right), i<k$ and therefore have an infinite intersection by property (i) of the $n$-optimal matrix.

For the higher successor case $\beta=\gamma+1, \alpha<\gamma$, we simulate this initial situation. By the inductive hypothesis pick a node

$$
r_{0} \in \bigcup_{i<k} \psi_{s_{i}, s_{0}}{ }^{\prime \prime}\left(t_{i}^{-} / \equiv_{m}\right)>s_{0},
$$

and let $r_{i}:=\psi_{s_{0}, s_{i}}\left(r_{0}\right)>s_{i}$ for $i<k$. We then know that $r_{i} \equiv_{m} t_{i}^{-}$, so $t_{i} / \equiv_{m}$ has elements above $r_{i}$. As a consequence $\bigcup_{i<k} \psi_{r_{i}, r_{0}}{ }^{\prime \prime}\left(t_{i} / \equiv_{m}\right)$ is infinite by the same argument as above and furthermore a subset of $\bigcup_{i<k} \psi_{s_{i}, s_{0}}{ }^{\prime \prime}\left(t_{i} / \equiv_{m}\right)$.

For the case where $\beta$ is a limit ordinal we choose $\gamma<\beta$ large enough, such that letting $q_{i}=t_{i}\left\lceil\gamma\right.$ for all $i, j<k$ we have $\psi_{q_{i}, q_{j}}\left(t_{i}\right)=t_{j}$. This is possible due to the transitivity of the coherent family. We also require $\alpha<\gamma$. The inductive hypothesis gives us a node

$$
r_{0} \in \bigcup_{i<k} \psi_{s_{i}, s_{0}}{ }^{\prime \prime}\left(q_{i} / \equiv_{m}\right) \subset T_{\gamma},
$$

which we copy to $r_{i}:=\psi_{s_{0}, s_{i}}\left(r_{0}\right)$. By this choice, we also have $r_{i} \equiv_{m} q_{i}$. We consider $u=\psi_{q_{i}, r_{0}}\left(t_{i}\right)$. By the commutativity of the coherent family this definition of $u$ is independent of the choice of $i<k$. But then

$$
\psi_{s_{0}, s_{i}}(u)=\psi_{r_{0}, r_{i}}(u)=\psi_{r_{0}, r_{i}} \circ \psi_{q_{i}, r_{0}}\left(t_{i}\right)=\psi_{q_{i}, r_{i}}\left(t_{i}\right)
$$

where the first equality follows from coherence, the second from the definition of $u$ and the third one from commutativity. So the property stated above right after the construction of $\equiv_{m}$ implies that $\psi_{s_{i}, s_{0}}\left(t_{i}\right) \equiv_{m} u$ since $r_{i} \equiv_{m} t_{i}$ for all $i<k$. This completes the proof of the Claim.

By virtue of the Claim we can pick for every tuple $\left(t_{0} / \equiv_{m}, \ldots, t_{k-1} / \equiv_{m}\right)$ of our antichain $A \subset \bigotimes_{i<k} S_{i}$ a node $u \in \bigcap_{i<k} \psi_{s_{i}, s_{0}}{ }^{\prime \prime}\left(t_{i} / \equiv_{m}\right)$ and collect all 
these nodes in a set $B$. Then $B$ is clearly an antichain of $T$ with the same cardinality as $A$. So we have shown that $T / \equiv_{m}$ is indeed a free tree.

Now for the second property. Let $\left(s_{0}, \ldots, s_{n-1}\right)$ be any sequence of nodes of some $T_{\alpha}$. We need to show that $\bigcap_{m<n} s_{m} / \equiv_{m}$ has a unique element. This is done by induction on $\alpha>0$. Starting with $\alpha=1$ we know that $\left(s_{m} / \equiv_{m}\right)=a_{i_{m}}^{k_{m}, m}$ (root) for some $i_{m}$ and $k_{m}$. So property (ii) of our $n$ optimal matrix is all we need here. For $\alpha=\gamma+1$ we assume that the classes $s_{m}^{-} / \equiv_{m}$ meet in a single node, say $r \in T_{\gamma}$. The set of elements of $s_{m} / \equiv_{m}$ which lie above $r$ is then just $a_{i_{m}}^{h_{m}(r), m}(r)$ and again property (ii) of the matrix proves the claim. In the limit case we once more use the transitivity of the coherent family. So let $\alpha$ be a limit and $\gamma<\alpha$ large enough such that $\psi_{q_{m}, q_{\ell}}\left(s_{m}\right)=s_{\ell}$ where we abbreviate $s_{m}\left\lceil\gamma=q_{m}\right.$. For the last time in this proof we use the commutativity of the coherent family: Let $r$ be the unique element of the intersection of the classes $q_{m} / \equiv_{m}$. Then $t=\psi_{q_{m}, r}\left(s_{m}\right)$ is well defined and independent of the choice of $m<n$. By the lifting property of the equivalence relations stated above, it follows from $q_{m} \equiv_{m} r$ that $s_{m} \equiv_{m} t$.

We now state an algebraic feature which distinguishes our method of decomposition as just carried out from other decompositions, namely that partial products of our decomposition are always rigid (cf. Remark 2.7).

Proposition 2.5. Let $T$ be a strongly homogeneous Souslin tree and assume that it has been decomposed into a product of $n$ free trees $S_{0}, \ldots, S_{n-1}$ by the procedure presented in the last proof. Then the product of less than $n$ pairwise distinct trees from the sequence $S_{0}, \ldots, S_{n-1}$ is a rigid Souslin tree.

Proof. It is clear that the product tree is Souslin and that it is sufficient to show rigidity only for the case of $n-1$ factors, where $n>2$. So assume that $R:=\bigotimes_{i<n-1} S_{i}$ admits the automorphism $\varphi^{\prime} \neq \mathrm{id}$ and derive a contradiction as follows.

Identifying $T$ and $R \otimes S_{n-1}$ we can lift $\varphi^{\prime}$ to an automorphism $\varphi=\varphi^{\prime} \otimes$ id of $T$. By Proposition 2.2 there is a countable ordinal $\alpha$ such that above level $T_{\alpha}$ the mapping $\varphi$ is given by a subfamily of the coherent family of $T$. As $\varphi^{\prime} \neq$ id there must be a node $\bar{s}=\left(s / \equiv_{0}, \ldots, s / \equiv_{n-1}\right) \in R$ such that $\varphi^{\prime}(\bar{s}) \neq \bar{s}$. We certainly can assume that $\operatorname{ht}_{R}(\bar{s})=\alpha$.

Pick an $\ell<n-1$ such that the $\ell$ th component $\bar{s}_{\ell}$ of $\bar{s}$ is not mapped to itself under $\varphi^{\prime}$. We fix a representative $s \in \bar{s}_{\ell}=s / \equiv_{\ell} \subset T_{\alpha}$ and let $q:=\varphi(s)$ in order to get $\varphi(r)=\psi_{s q}(r)$ for all (immediate) successors of $s$. This is where we will find a contradiction.

In the construction above we have associated to the class $s / \equiv_{\ell}$ a natural number $h(s, \ell)$ which defined the index of the partition that was used to extend $\equiv_{\ell}$ to the successors of the members of the class $s / \equiv_{\ell}$. The same 
holds for $q=\varphi(s)$, but as $q \not \equiv \ell s$ we have $h(q, \ell) \neq h(s, \ell)$. This implies that $\equiv_{\ell}$ for immediate successors of $s$ and of $q$ is formed by virtue of different partitions of column $\ell$ of our partition matrix. Fix any immediate successor $r$ of $s$. Since $\varphi^{\prime}$ is assumed to be a well-defined mapping, $\varphi$ should map the intersection $\bigcap_{i<n-1} r / \equiv_{i}$ onto the set $\bigcap_{i<n-1} \varphi(r) / \equiv_{i}$. In particular, the equality

$$
\psi_{s q}^{\prime \prime}\left(T(s) \cap \bigcap_{i<n-1} r / \equiv_{i}\right)=T(q) \cap \bigcap_{i<n-1} \psi_{s q}(r) / \equiv_{i}
$$

should be true. But this is not the case, because, by $n$-optimality of the matrix of partitions, the left-hand side of the above equality intersects every

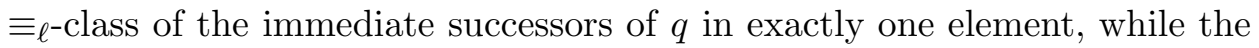
right-hand side is a subset of such an $\equiv_{\ell^{-}}$class and at the same time an infinite set.

In the next section we will find corollaries of Theorem 2.4 in a similar vein. I especially mention Lemma 3.6 which states that forcing with such a partial product (as above) turns the complementary partial product, which was rigid in the ground model, into a strongly homogeneous Souslin tree in the generic extension.

The following complements Theorem 2.4 .

TheOREM 2.6. Every $\aleph_{0}$-branching, strongly homogeneous Souslin tree $T$ is (isomorphic to) the tree product of $n$ strongly homogeneous Souslin trees for any given natural number $n>0$.

Proof. This is just a simpler variant of the construction in the proof of Theorem 2.4 where we use only the first row of the matrix of partitions (or just any bijection between $\omega^{n}$ and $\omega$ ). It is then easy to verify that the coherent family of $T$ descends to the factor trees thus obtained and renders them strongly homogeneous.

REMARK 2.7.

(i) Though, of course, not every tree product of two strongly homogeneous Souslin trees is Souslin again (e.g. take $T \otimes T$ ), there is a converse to the last theorem: If $S$ and $T$ are strongly homogeneous Souslin trees and the tree product $S \otimes T$ satisfies the c.c.c., then $S \otimes T$ is a strongly homogeneous Souslin tree as well.

(ii) We see that there are two essentially distinct ways to decompose a strongly homogeneous tree into (at least three) free factors. An application of Theorem 2.6 to decompose a given strongly homogeneous Souslin tree $T$ into $\ell$ strongly homogenous factors $S_{0}, \ldots, S_{\ell-1}$ followed by an $\ell$-fold application of the procedure used in the proof of Theorem 2.4 to decompose the tree $S_{k}$ into $m_{k}$ free trees $R_{i}^{k}$ for 
$0 \leq i<m_{k}$ never results in the same decomposition as directly using the proof of Theorem 2.4 to decompose $T$ into $\sum_{k=0}^{\ell-1} m_{k}$ free factors. The partial products of the latter decomposition are all rigid by Proposition 2.5 while the first also has partial products that are strongly homogeneous.

3. Separating high degrees of rigidity. In this section we review several families of rigidity notions for Souslin trees, all of them weaker than freeness. These definitions (except for that of an $n$-free Souslin tree) are all taken from [FH09]. Most of these definitions refer to the technique of forcing applied with a Souslin tree as the forcing partial order. We do not review forcing here. But recall that forcing with a Souslin tree always assumes the inverse order on the tree (i.e., trees grow downwards when considered as forcing partial orders, the root is the maximal element, etc.) and adjoins a cofinal branch.

This section is divided into five short subsections. The first two introduce the rigidity notions to be considered and the last three state many and prove some separations between them. We only give proofs that either are elementary or use the proof of the Decomposition Theorem 2.4.

3.1. Parametrized freeness. Considering the definition of the property of being free for Souslin trees it is natural to ask whether or not it makes any difference if the number of factors in the tree products that are required to be Souslin is bounded. This leads to the following definition which we rightaway connect to the definition of being $n$-fold Souslin off the generic branch met in [FH09].

DeFinition 3.1. Let $n$ be a positive natural number.

(a) We say that a Souslin tree $T$ is $n$-free if for every subset $P$ of size $n$ of some level $T_{\alpha}, \alpha<\omega_{1}$, the tree product $\bigotimes_{s \in P} T(s)$ satisfies the c.c.c.

(b) A Souslin tree is said to be $n$-fold Souslin off the generic branch if for any sequence $\vec{b}=\left(b_{0}, \ldots, b_{n-1}\right)$ generic for the $n$-fold forcing product of (the inverse partial order of) $T$ and any node $s \in T \backslash \bigcup_{i \in n} b_{i}$, the subtree $T(s)$ of all nodes of $T$ above $s$ is a Souslin tree in the generic extension $M[\vec{b}]$ (which amounts to requiring that the adjunction of $\vec{b}$ does not collapse $\omega_{1}$ and preserves the c.c.c. of the $\left.T(s), s \notin \bigcup b_{i}\right)$.

It is easy to see that a 2 -free Souslin tree or a tree which is Souslin off the generic branch cannot be decomposed as the product of two Souslin trees. And this common feature is no coincidence.

Proposition 3.2. For a positive natural number $n$ and a normal Souslin tree $T$ the following statements are equivalent. 
(a) $T$ is $n$-fold Souslin off the generic branch.

(b) $T$ is $(n+1)$-free.

Proof. We start with the implication (b) $\Rightarrow(\mathrm{a})$. Assume that $T$ is $n+1$ free and let $\vec{b}=\left(b_{0}, \ldots, b_{n-1}\right)$ be generic for $T^{\otimes n}$, the $n$-fold tree product of $T$ with itself. Choose $\alpha<\omega_{1}$ large enough such that the nodes $t_{i}:=b_{i}(\alpha)$ are pairwise incompatible. Finally, pick a node $t_{n} \in T_{\alpha}$ distinct from all the $b_{i}(\alpha)$. By our freeness assumption on $T$, the product tree $\bigotimes_{i \in n+1} T\left(t_{i}\right)$ satisfies the countable chain condition. But then $M[\vec{b}] \vDash$ " $T\left(t_{n}\right)$ is Souslin" by a standard argument concerning chain conditions in forcing iterations. Now it is easy to see that $T$ is $n$-fold Souslin off the generic branch.

For the other direction we inductively show that $T$ is $m$-free for $m \leq n+1$, assuming that $T$ is $n$-fold Souslin off the generic branch. The inductive claim is trivial for $m=1$. So let $m \geq 1$ and let $s_{0}, \ldots, s_{m}$ be pairwise distinct nodes of the same height. Then for any generic sequence $\vec{b}=\left(b_{0}, \ldots, b_{m-1}\right)$ for $\bigotimes_{i \in m} T\left(s_{i}\right)$ we know that $T\left(s_{m}\right)$ is Souslin in the generic extension $M[\vec{b}]$. Finally the two-step iteration $\bigotimes_{i \in m} T\left(s_{i}\right) * \check{T}\left(s_{m}\right)$ is isomorphic to $\bigotimes_{i \in m+1} T\left(s_{i}\right)$ and satisfies the countable chain condition.

This proposition implies that a free tree $T$ is also free off the generic branch in the sense that in the generic extension obtained by adjoining a cofinal branch $b$ through $T$, for every node $t \in T \backslash b$, the tree $T(t)$ is still free.

3.2. Further types of rigidity. In Sections 1-4 of [FH09] different notions of rigidity for Souslin trees are collected: (ordinary) rigidity, total rigidity and the unique branch property and their absolute counterparts, where absoluteness refers to forcing extensions obtained by adjoining a generic branch to the Souslin tree under consideration. In this context also the stronger notion of being ( $n$-fold) Souslin off the generic branch is introduced, which we already considered in the last section.

DeFinition 3.3.

(a) A Souslin tree $T$ is called $n$-absolutely rigid if $T$ is a rigid tree in the generic extension obtained by forcing with $T^{n}$ (or equivalently $T^{\otimes n}$ ).

(b) A Souslin tree is totally rigid if the trees $T(s)$ and $T(t)$ are nonisomorphic for all pairs of distinct nodes $s$ and $t$ of $T$. It is $n$-absolutely totally rigid if it is totally rigid after forcing with $T^{n}$.

(c) A Souslin tree $T$ has the unique branch property (UBP) if forcing with $T$ adjoins only a single cofinal branch to $T$. For $n>0$ we say that $T$ has the $n$-absolute $U B P$ if forcing with $T^{n+1}$ adjoins exactly $n+1$ cofinal branches to $T$.

Fuchs and Hamkins prove implications as well as some independencies between these rigidity notions. They also give in [FH09, Section 4] a diagram 
of implications between the degrees of rigidity that we have approximately reconstructed here for the convenience of the reader.

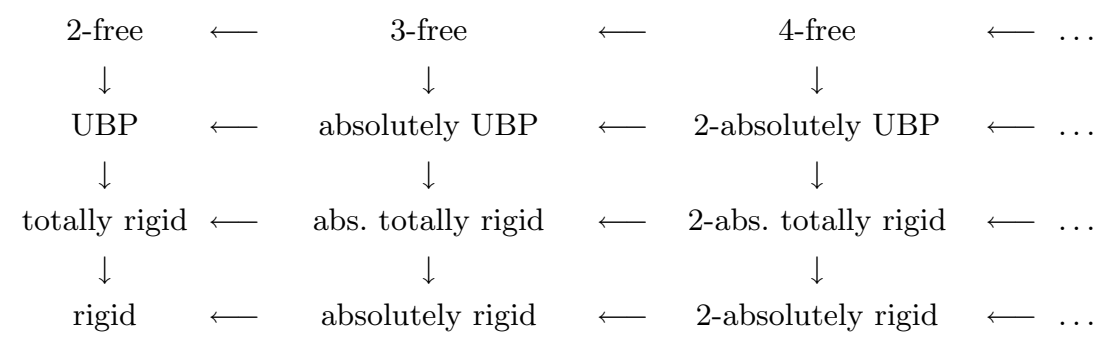

Diagram 1. Implications between degrees of rigidity for Souslin trees

Fuchs and Hamkins show that the part of the diagram to the left and below "absolutely UBP" is complete in the sense that there are no further general implications between these rigidity properties. They ask whether the rest of the diagram is complete as well (cf. [FH09, Question 4.1]). We will show (resp. state) below that there are neither implications from left to the right (including downward diagonals, cf. Corollaries 3.5 and 3.7), nor from the second to the top row (Theorem 3.9).

REMARK 3.4. Using a standard $\diamond$-construction scheme for a Souslin tree (e.g., cf. [FH09, Section 2]) it is not hard to construct a Souslin tree $T$ with the following two features:

- On each level $T_{\alpha}$ no two distinct nodes have the same number of immediate successors. So in particular $T$ is $n$-absolutely totally rigid for every $n \in \omega$.

- The substructure $R$ of $T$ obtained by restricting the supporting set to the nodes on the limit levels of $T$ plus the root, is a homogeneous Souslin tree. Then in a generic extension obtained by forcing with $T$ there are many cofinal branches in $R$ and each of them gives rise to a cofinal branch of $T$, which is thus not a UBP tree. (In fact, every $\aleph_{0^{-}}$ splitting Souslin tree can be extended to an $n$-absolutely totally rigid Souslin tree by inserting new successor levels such that any two nodes of the same height have a different number of immediate successors.)

This shows that in Diagram 1 there can be no arrows that point upwards from the two bottom rows. So the only question left open is whether there should be any more arrows between the two bottom rows, but a similar construction as the one alluded to above should also eliminate those.

3.3. Distinct degrees of freeness. Our next corollary of (the proof of) Theorem 2.4 gives the separation of the finite degrees of freeness, i.e., 
it shows that the family of parametrized freeness conditions is properly increasing in strength.

COROLlary 3.5. If there is a strongly homogeneous Souslin tree, then there is a tree that is $n$-free, but not $n+1$-free.

Proof. Let the strongly homogeneous Souslin tree $T$ be decomposed as the tree product of $n$ free trees $S_{i}$ for $i<n$ as in the proof of Theorem 2.4. We show that the tree sum of the factors,

$$
R:=\bigoplus_{i<n} S_{i}
$$

is an $n$-free but non- $n+1$-free Souslin tree. The Claim used in the proof of Theorem 2.4 remains true in the following variant:

Claim ${ }^{\prime}$. For any pair of sequences $\left(s_{0}, \ldots, s_{n-1}\right)$ in $T_{\alpha}$ and $t_{i}>s_{i}$ in $T_{\beta}$ and any sequence $m: n \rightarrow n$ the intersection

$$
\bigcap_{i<n} \psi_{s_{i}, s_{0}}{ }^{\prime \prime} t_{i} / \equiv_{m(i)}
$$

is not empty.

Modulo the obvious changes in the notation, the proof of the Claim' remains completely the same as before, exploiting the $n$-optimality of the matrix. And also with the same argument as above we can construct an antichain of $T$ from any given antichain of $R$ maintaining the cardinality. So $R$ is $n$-free.

We now argue that $R$ is not $n$-fold Souslin off the generic branch.

If $b_{i}$ is a cofinal branch through $S_{i}$ then in the generic extension obtained by adjoining $\vec{b}=\left(b_{0}, \ldots, b_{n-1}\right)$, the strongly homogeneous tree $T$ has a cofinal branch as well, thus destroying the Souslinity of all subtrees of $R$.

3.4. Freeness and absolute rigidity. In this section we improve upon the result of the last one by showing that $n$-freeness of a tree does not even imply $(n-1)$-absolute rigidity.

Lemma 3.6. Let the strongly homogeneous Souslin tree $T$ be decomposed as the tree product of $n$ free trees $S_{i}$ for $i<n$ as in the proof of Theorem 2.4. Let $\{a, b\}$ be a partition of the set $n$ with $a, b \neq \emptyset$ and set $\mathbb{P}:=\bigotimes_{i \in a} S_{i}$ and $R:=\bigotimes_{i \in b} S_{i}$. Then

$$
\vDash_{\mathbb{P}} \text { " } \check{R} \text { is strongly homogeneous". }
$$

Proof. We adopt the notation from the statement of the lemma and argue inside the generic extension obtained by adjoining a generic branch $c$ to the Souslin tree $\mathbb{P}$. Then we have the natural isomorphism $\rho: R \cong c \otimes R \subset T$. Denote the canonical projection $T \rightarrow R$ by $\pi$. 
We define the tree isomorphisms $\varphi_{r s}$ (members of the coherent family of $R$ in the generic extension) for nodes $r$ and $s$ of $R_{\alpha}, \alpha<\omega_{1}$, from the members $\psi_{\rho(r) \rho(s)}$ of the coherent family of $T$. For this, we refer to the maps $h(\cdot, j): T \rightarrow \omega$ used in the construction of the t.e.r.s $\equiv_{j}$ in the proof of Theorem 2.4. We collect them and define $h: T \rightarrow \omega^{|b|}$ by simply concatenating the values $h(t, j)$ for $t \in T$ and $j \in b$.

If $r, s \in R_{\alpha}$ and $h(\rho(r))=h(\rho(s))$, then we let

$$
\varphi_{r s}:=\pi \circ \psi_{\rho(r) \rho(s)} \circ \rho: R(r) \rightarrow R(s) .
$$

It follows from the fact that the $n$-optimal partition matrices are transported between the (sets of immediate successors of the) nodes by the members $\psi_{t u}$ of the coherent family of $T$ that this definition is sound and indeed yields an isomorphism.

Now let $r, s \in R_{\alpha}$ with $h(\rho(r)) \neq h(\rho(s))$. In order to define $\varphi_{r s}$ we compose the tree isomorphisms that we have already defined for the immediate successors of $r$ and $s$. For every direct successor $u \in \operatorname{succ}(r)$ there is exactly one $v \in \operatorname{succ}(s)$ with $h(\rho(u))=h(\rho(v))$. This follows from the $n$-optimality of the partition matrix. Let $\varphi_{r s}(u)$ be just this $v$. If $x$ is a non-immediate successor of $r$, then first find the immediate successor $u$ of $r$ below $x$ and the image $v=\varphi_{r s}(u)$, and set

$$
\varphi_{r s}(x):=\varphi_{u v}(x) .
$$

It remains to prove that the family of tree isomorphisms just defined is coherent, commutative and transitive. Commutativity and coherence are inherited from the coherent family of $T$. (Note that $\varphi_{r s}(x)=y$ implies that $h(\rho(x))=h(\rho(y))$, so the two cases do not interfere.) As for transitivity, let $x, y \in R_{\lambda}$ for some countable limit ordinal $\lambda$. Then by the transitivity of the family of the $\psi_{t u}$ for $T$ there are $t<\rho(x)$ and $u<\rho(y)$ with $\psi_{t u}(\rho(x))=$ $\rho(y)$. But then $t$ and $u$ lie in $b \otimes R$, so there are $r, s \in R$ such that $\rho(r)=t$ and $\rho(s)=u$ and thus $\varphi_{r s}(x)=y$.

So, e.g. in the case $n=2$, forcing with one free tree does not only destroy the freeness of another one, but even turns the latter into a strongly homogeneous Souslin tree, i.e., it adjoins many generic automorphisms.

Corollary 3.7. Let $n>1$. If there is a strongly homogeneous Souslin tree, then there is an n-free tree which is not $(n-1)$-absolutely rigid.

Proof. We fix $n>1$ and use the tree $R$ from the proof of Corollary 3.5 obtained from a strongly homogeneous tree $T$ as the tree sum $R=\bigoplus_{i<n} S_{i}$ of the free factors $S_{i}, i<n$, of $T$. From Corollary 3.5 we know that $R$ is $n$-free.

To show that $R$ is not $(n-1)$-absolutely rigid we refer to Lemma 3.6 . It follows directly from the case that $a=n \backslash\{i\}$ for some $i<n$ that $R$ 
is not rigid in the generic extension obtained by adjoining a cofinal branch through the trees $S_{j}$ for $j<n$ and $j \neq i$. But this generic extension can also be reached by forcing with $R^{\otimes n-1}$.

3.5. Freeness and the unique branch property. We start with an easy result deduced from the elementary properties of finitely free trees for the second column of the diagram.

Proposition 3.8. If there is a 3-free Souslin tree, then there is also a Souslin tree which has the UBP and is not 2-free.

Proof. Let $T$ be 3 -free and pick distinct nodes $s, t \in T$ of the same height. We show that the Souslin tree $S=T(s) \otimes T(t)$ has the UBP. Let $b \otimes c$ be a generic, cofinal branch in $S$ (we view $b$ and $c$ as trees). By the 2 -fold Souslinity off the generic branch of $T$, every tree of the form $T(r)$ with $r \in T \backslash(b \cup c)$ is Souslin in the generic extension by $b \otimes c$. On the other hand, if there were a second cofinal branch through $S$ in the generic extension, then one of its components would have to pass through such a node $r \notin b \cup c$, which yields a contradiction.

To prove that $S$ is not 2-free, let $u>s$ and $v, w>t$ be of the same height, where $v \neq w$. Then

$$
S(u, v) \otimes S(u, w) \cong T(u) \otimes T(v) \otimes T(u) \otimes T(w)
$$

has an uncountable antichain, because it has the square of the Souslin tree $T(u)$ as a factor.

This result cannot be improved by simply requiring $T$ to be free, because by iterating the forcing with a tree product of two factors $n+1$ times, we adjoin at least $2^{n}$ cofinal branches.

We do have the following non-implication result for the $n$-absolute UBP and 2 -freeness under the stronger assumption of $\diamond$.

Theorem 3.9. Assume $\diamond$. Then there is a Souslin tree which is not 2 -free but has the $n$-absolute $U B P$ for all $n \in \omega$.

The methods of proof for this theorem lie beyond the scope of this paper. It uses ideas from [FH09] and [SF09]. A proof sketch can be found in [SF08, Theorem 1.6.3].

3.6. Further directions. As a closing remark we mention how Diagram 1, which captures the implications between four families of rigidity notions and implications between them, could possibly be extended.

Real rigidity: In [AS85] two Aronszajn trees are called really different if there is no isomorphism between any of their restrictions to some club set of levels. In this vein, we could call a Souslin tree really rigid if all of its restrictions to club sets of levels are rigid. This property is clearly stronger 
than ordinary rigidity yet independent of total rigidity (cf. Remark 3.4) and is implied by the unique branching property. Also the variant of real, total rigidity and the $n$-absolute versions of real rigidity and of real, total rigidity could be considered.

Self-specializing trees: A normal tree $T$ of height $\omega_{1}$ is called special if there is a countable family $\left(A_{n}\right)_{n \in \omega}$ of antichains of $T$ that covers all of $T$. As $T$ is uncountable, one of the $A_{n}$ has to be uncountable as well, so a special tree $T$ is not Souslin. On the other hand, every branch of $T$ meets each antichain $A_{n}$ in at most one node and is therefore countable.

A self-specializing tree is a Souslin tree $T$ that specializes itself by forcing a generic branch $b$ through it, i.e., in the generic extension obtained by adjoining $b$ to the universe, the tree $T \backslash b$ is special. Self-specializing trees can be found in models of $\diamond$. They are UBP: a second cofinal branch in $T$ would prevent $T \backslash b$ from being special. But of course they are not Souslin off the generic branch, and they can neither be 2-absolutely really rigid nor absolutely UBP, because forcing with a special tree collapses $\omega_{1}$, and in this second generic extension the limit levels of $T$ form an $\aleph_{0}$-splitting tree of countable height which must be homogeneous by a result of Kurepa (cf. [Kur35, p. 102]).

Now let us call a Souslin tree $T n$-self-specializing if it is $n$-free (i.e. $(n-1)$-fold Souslin off the generic branch) and forcing a generic branch $\vec{b}$ through $T^{n}$ makes $T \backslash \tilde{b}$ special where $\tilde{b}$ is the set of components of the elements of $\vec{b}$. It is not yet verified but seems quite plausible that one can construct an $n$-self-specializing tree under $\diamond$. In the implication diagram its place could be between $n$-free and $(n-1)$-absolutely UBP, yet it is stronger than both of these properties. And there would be no horizontal implications, for an $n$-self-specializing tree is neither $(n-1)$-self-specializing nor $(n+1)$-self-specializing.

As is clear from the outset, adding these families to Diagram 1 results in a far more complicated directed graph which is in particular non-planar. We leave such considerations for future work.

Acknowledgments. Thanks are due to Piet Rodenburg for pointing out a flaw in an earlier version of the proof of Lemma 1.2 .

\section{References}

[AS85] U. Abraham and S. Shelah, Isomorphism types of Aronszajn trees, Israel J. Math. 50 (1985), 75-113.

[AS93] - - - $A \Delta_{2}^{2}$ well-order of the reals and incompactness of $L\left(Q^{\mathrm{MM}}\right)$, Ann. Pure Appl. Logic 59 (1993), 1-32. 
[DJ74] K. J. Devlin and H. Johnsbråten, The Souslin Problem, Lecture Notes in Math. 405, Springer, 1974.

[FH09] G. Fuchs and J. D. Hamkins, Degrees of rigidity for Souslin trees, J. Symbolic Logic 74 (2009), 423-454.

[Jec03] T. Jech, Set Theory, 3rd ed., Springer, 2003.

[Jen] R. B. Jensen, The generic Kurepa hypothesis $I+I I$, handwritten notes.

[Kun80] K. Kunen, Set Theory. An Introduction to Independence Proofs, North-Holland, 1980 .

[Kur35] Đ. Kurepa, Ensembles ordonnés et ramifiés, Publ. Math. Univ. Belgrade 4 (1935), 1-138; republished in: A. Ivić et al. (eds.), Selected Papers of Đuro Kurepa, SANU, 1996.

[Lar99] P. Larson, An $\mathbb{S}_{\max }$ variation for one Souslin tree, J. Symbolic Logic 64 (1999), 81-98.

[LT02] P. Larson and S. Todorčević, Katětov's problem, Trans. Amer. Math. Soc. 354 (2002), 1783-1791.

[SF08] G. Scharfenberger-Fabian, Subalgebras of small Souslin algebras and maximal chains in Souslin algebras, PhD thesis, Freie Univ. Berlin, 2008; www.diss.fuberlin.de/diss/receive/FUDISS_thesis_000000005206

[SF09] —, Souslin algebra embeddings, Arch. Math. Logic, to appear; www.arxiv.org/ abs/0904.0113.

[SZ99] S. Shelah and J. Zapletal, Canonical models for $\aleph_{1}$-combinatorics, Ann. Pure Appl. Logic 98 (1999), 217-259.

[Tod84] S. Todorčević, Trees and linearly ordered sets, in: Handbook of Set-Theoretic Topology, Chap. 6, North-Holland, 1984, 235-294.

Gido Scharfenberger-Fabian

Institute of Mathematics and Computer Sciences

Ernst-Moritz-Arndt-University

Walther-Rathenau-Strasse 47

17487 Greifswald, Germany

E-mail: gsf@uni-greifswald.de

Received 14 October 2009;

in revised form 2 June 2010 